\title{
Proceedings
}

\section{Retrieving the True Masses of Gravitational Wave Sources ${ }^{+}$}

\author{
Xian Chen ${ }^{1,2, *(1)}$ and Zhefeng Shen ${ }^{1}$ \\ 1 Astronomy Department, School of Physics, Peking University, Beijing 100871, China \\ 2 Kavli Institute for Astronomy and Astrophysics at Peking University, Beijing 100871, China \\ * $\quad$ Correspondence: xian.chen@pku.edu.cn; Tel.: +86-10-6275-5391 \\ + Presented at the meeting Recent Progress in Relativistic Astrophysics, Shanghai, China, 6-8 May 2019.
}

Received: 26 June 2019; Accepted: 15 July 2019; Published: 17 July 2019

\begin{abstract}
Gravitational waves (GWs) encode important information about the mass of the source. For binary black holes (BBHs), the templates that are used to retrieve the masses normally are developed under the assumption of a vacuum environment. However, theories suggest that some $\mathrm{BBHs}$ form in gas-rich environments. Here, we study the effect of hydrodynamic drag on the chirp signal of a stellar-mass BBH and the impact on the measurement of the mass. Based on theoretical arguments, we show that the waveform of a $\mathrm{BBH}$ in gas resembles that of a more massive $\mathrm{BBH}$ residing in vacuum. The effect is important for those GW sources in the band of space-borne detectors but negligible for those in ground-based ones. Furthermore, we carry out a matched-filtering search of the best fitting parameters. We find that the best-fit chirp mass could be significantly greater than the real mass when the gas effect is ignored. Our results have important implications for the future joint observation of BBHs using both ground- and space-based detectors.
\end{abstract}

Keywords: high energy astrophysics; black holes; neutron stars; gravitational waves

\section{Introduction}

Measuring the mass of a gravitational wave (GW) source is an old but difficult problem because the observable is not mass, but the phase and amplitude of GWs. A model is needed to translate the observables to the mass. However, the standard model that is used in the current GW observations often neglects the astrophysical factors, which could affect the dynamical evolution of the source or the propagation of the GWs. Consequently, the standard model could potentially misinterpret the GW signal.

For example, redshift is such a factor [1,2]. It stretches the waveform so that a low-mass source at high redshift looks identical to a massive one at low redshift. Such a "mass-redshift" degeneracy has become a serous issue because the Laser Interferometer Gravitational-wave Observatory (LIGO) and the Virgo detectors have detected seemly over-massive black holes (BHs) [3,4], which are two to three times bigger than those $\mathrm{BHs}$ previously detected in X-ray binaries [5,6]. On one hand, the high masses may be real and reflect the peculiarity of the environment in which the BHs form [7-12]. Alternatively, the BHs may be intrinsically small but appear more massive due to a high redshift.

The high redshift could be explained in two astrophysical scenarios. One possibility is that binary $\mathrm{BHs}$ (BBHs) coalesce at high cosmological redshift and get strongly lensed by foreground galaxies or galaxy clusters $[13,14]$. This scenario, although possible for a small number of of BBHs, could not account for all the massive BHs detected so far by LIGO/Virgo $[3,15]$. Another possibility is that BBHs merge in places very close to supermassive black holes (SMBHs), so that both the Doppler and gravitational redshift become significant $[2,16,17]$. The problem with this scenario is that the event 
rate is difficult to estimate because the stellar distribution around SMBHs is not well constrained by observations [2].

Is there a way of distinguishing, on a one-to-one basis, the redshifted BBHs from the intrinsically massive ones? It is difficult using ground-based detectors. The corresponding signals usually last no more than one second, too short to reveal any signature of gravitational lensing or a nearby SMBH. However, with a space-borne GW detector, such as the Laser Interferometer Space Antenna (LISA [18]), the answer would be different. Being sensitive to milli-Hertz ( $\mathrm{mHz}$ ) GWs, LISA could detect $\mathrm{BBHs}$ at a much earlier evolutionary stage, weeks to millenniums before they enter the LIGO/Virgo band [19-21]. The corresponding signals could be as long as the lifetime of LISA, about 4-5 years. Earlier studies showed that, if a BBH is strongly lensed, LISA could detect multiple images of the source $[22,23]$ or, in some rare cases, detect a shift of the GW phase caused by the wave effect of gravitational lensing [24,25]. If, on the other hand, a BBH is close to a SMBH, LISA could detect a distortion of the waveform caused by either the orbital motion of the binary around the SMBH [26-30] or the tidal force of the SMBH $[27,31-33]$.

Besides redshift, are there other astrophysical factors that could affect the measurement of the masses of GW sources? In this article, we show that the presence of gas around BBHs could also lead to an overestimation of the masses. Investigating this scenario is important because in many theoretical models the merger of stellar-mass BBHs is driven by gas (e.g., [9-12,34-38]).

\section{The Effect of Gas on the Measurement of Mass}

The waveform of a merging $\mathrm{BBH}$ can be divided into three parts, corresponding to the inspiral, merger, and ringdown phases [39]. During the inspiral phase, the information of mass is encoded in the GW frequency $(f)$ and the time derivative of it $(\dot{f})$. For example, consider a BBH whose BH masses are $m_{1}$ and $m_{2}$ (we assume $m_{1} \geq m_{2}$ ). If the binary is in vacuum, the following quantity

$$
\mathcal{M}:=\frac{c^{3}}{G}\left(\frac{5 f^{-11 / 3} \dot{f}}{96 \pi^{8 / 3}}\right)^{3 / 5}
$$

is equivalent to

$$
\mathcal{M}=\frac{\left(m_{1} m_{2}\right)^{3 / 5}}{\left(m_{1}+m_{2}\right)^{1 / 5}}
$$

according to the Newtonian approximation [40], where $G$ is the gravitational constant and $c$ the speed of light. This quantity has the dimension of mass and uniquely determines how the GW frequency increases with time. It is known as the "chirp mass".

Gas could make BBHs shrink more rapidly by imposing a hydrodynamical drag on each BH [41]. As a result, the observed $\dot{f}$ would be bigger than that in the vacuum case. Without knowing the gas effect, an observer is likely to apply the vacuum model, i.e., Equation (1), to the observed $f$ and $\dot{f}$. The derived mass, which we call $\mathcal{M}_{0}$, will be bigger than the real chirp mass.

To see this effect more clearly, we first denote the value of $\dot{f}$ in the vacuum model as $\dot{f}_{\mathrm{gw}}$ and that in the gas model as $\dot{f}_{\text {gas }}$. Furthermore, we define the semi-major axis of a BBH as $a$ and the shrinking rate due to GW radiation as $\dot{a}_{\mathrm{gw}}$. Then, we can express the shrinking timescale due to GW radiation as $T_{\mathrm{gw}}:=a /\left|\dot{a}_{\mathrm{gw}}\right|$. Now, suppose the presence of gas causes the binary to shrink at an additional rate of $\dot{a}_{\text {gas }}$, we can write the gas-drag timescale as $T_{\text {gas }}:=a /\left|\dot{a}_{\text {gas }}\right|$. Let us further assume for simplicity a circular binary, so that the GW frequency is $f=\pi^{-1} \sqrt{G\left(m_{1}+m_{2}\right) / a^{3}}$. From the last formula, we find $\dot{f}_{\text {gas }}=\left(1+T_{\mathrm{gw}} / T_{\text {gas }}\right) \dot{f}_{\mathrm{gw}}$. Substituting $\dot{f}_{\text {gas }}$ in Equation (1) for $\dot{f}$, we find that the "observable" mass is no long the intrinsic chirp mass, but

$$
\mathcal{M}_{o}=\left(1+T_{\mathrm{gw}} / T_{\text {gas }}\right)^{3 / 5} \mathcal{M}
$$

Interestingly, this mass could be much bigger than the real mass when $T_{\text {gas }} \ll T_{\text {gw }}$. 
Now, we compare the values of $T_{\text {gas }}$ and $T_{\mathrm{gw}}$. For circular binary and in the Newtonian approximation, the GW radiation timescale can be calculated with

$$
\begin{aligned}
T_{\mathrm{gw}} & :=\frac{a}{\left|\dot{\mathrm{g}}_{\mathrm{gw}}\right|}=\frac{5}{64} \frac{c^{5} a^{4}}{G^{3} m_{1} m_{2} m_{12}} \\
& \simeq \frac{9.1 \times 10^{3}}{q(1+q)^{-1 / 3}}\left(\frac{m_{1}}{10 M_{\odot}}\right)^{-5 / 3}\left(\frac{f}{3 \mathrm{mHz}}\right)^{-8 / 3} \text { years }
\end{aligned}
$$

(from [42]), where $q:=m_{1} / m_{1}$ is the mass ratio of the binary and $m_{12}:=m_{1}+m_{2}$. We are scaling the $\mathrm{GW}$ frequency to $\mathrm{mHz}$ because the corresponding semi-major axis is about

$$
a=\left(\frac{G m_{12}}{\pi^{2} f^{2}}\right)^{1 / 3} \simeq 0.0021\left(\frac{m_{12}}{20 M_{\odot}}\right)^{1 / 3}\left(\frac{f}{3 \mathrm{mHz}}\right)^{-2 / 3} \mathrm{AU} .
$$

BBHs with such a semi-major axis could have a gas-drag timescale as short as $T_{\text {gas }} \simeq 10^{3}$ years according to the earlier studies of the BBHs in gaseous environments (e.g., [10]). It is worth noting that $T_{\text {gas }}$ is a function of gas density and hence could be even shorter in the most gas-rich environment, such as the innermost part of the accretion disk around a SMBH or the common envelope surrounding a binary [12,34]. From the timescales derived above, we find that for LISA BBHs ( $f \sim 1 \mathrm{mHz})$ it is possible that $T_{\text {gas }} \ll T_{\text {gw }}$. For LIGO/Virgo BBHs $(f \sim 10 \mathrm{~Hz})$, gas drag is no longer important because the GW radiation timescale, according to Equation (5), is too short.

To be more quantitative, take $T_{\text {gas }}=10^{3}$ years and $T_{\mathrm{gw}}=10^{4}$ years for example. We have $T_{\mathrm{gw}} / T_{\text {gas }}=10$. According to Equation (3), one would overestimate the mass by a factor of 4.2 if the gas effect is ignored. In this case, a BBH with $m_{1}=m_{2}=10 M_{\odot}\left(\mathcal{M} \simeq 8.7 M_{\odot}\right)$ would appear to have a chirp mass of $\mathcal{M}_{0} \simeq 37 M_{\odot}$. In other words, from LISA waveform, it seems that two $42 M_{\odot}$ BHs are merging.

\section{Matched Filtering and Parameter Estimation}

In practice, LISA employs a technique called the "matched filtering" to estimate the parameters of a GW source [43]. In this technique, the similarity of two waveforms, say $h_{1}(t)$ and $h_{2}(t)$, is quantified by the "fitting factor" (FF), defined as

$$
\mathrm{FF}=\frac{\left\langle h_{1} \mid h_{2}\right\rangle}{\sqrt{\left\langle h_{1} \mid h_{1}\right\rangle\left\langle h_{2} \mid h_{2}\right\rangle}},
$$

where $\left\langle h_{1} \mid h_{2}\right\rangle$ denotes an inner product, which can be calculated with

$$
\left\langle h_{1} \mid h_{2}\right\rangle=2 \int_{0}^{\infty} \frac{\tilde{h}_{1}(f) \tilde{h}_{2}^{*}(f)+\tilde{h}_{1}^{*}(f) \tilde{h}_{2}}{S_{n}(f)} d f .
$$

In the last equation, the tilde symbols stand for the Fourier transformation and the stars stand for the complex conjugation. The quantity $S_{n}(f)$ is the spectral noise density of LISA (see details in [17]). An exact match, in principle, would mean $\mathrm{FF}=1$.

In reality, noise exists and consequently FF is not unity even when $h_{1}$ and $h_{2}$ are identical. A more practical definition of "match" is that $\langle\delta h \mid \delta h\rangle<1$, where $\delta h:=\tilde{h}_{1}(f)-\tilde{h}_{2}(f)$ [44]. There is a close relationship between $\langle\delta h \mid \delta h\rangle, \mathrm{FF}$, and the signal-to-noise ratio (SNR) defined as $\rho^{2}:=\langle h \mid h\rangle$, which could help with simplifying our calculation. We note that, in GW observations, often we are in the situation where $h_{1} \simeq h_{2}$. Therefore, we have $\rho^{2} \simeq\left\langle h_{1} \mid h_{1}\right\rangle \simeq\left\langle h_{2} \mid h_{2}\right\rangle$. Using the last equation, we find that the condition for match is equivalent to

$$
\text { FF }>1-1 /\left(2 \rho^{2}\right) .
$$


Since LISA will claim a detection when $\rho \simeq 10[20,45]$, only those temples with $F F>0.995$ are acceptable.

In our particular problem, $h_{1}(t)$ is the GW signal from a BBH embedded in a gaseous environment, and $h_{2}(t)$ is the waveform template that we use to match with $h_{1}(t)$ and extract physical parameters. To prepare a template bank for $h_{2}(t)$, a model is needed. Thus far, only the vacuum model has been considered in the literature. In the following, we show that, even though the vacuum model is an incorrect one, the resulting FF could still be very high. Consequently, using this model will confuse the estimation of the mass of a BBH.

In this work, we compute the waveforms using

$$
h(t)=\frac{A m_{1} m_{2}}{a(t)} \cos \phi(t),
$$

where $A$ is a normalization factor depending on the source distance but not important for matched filtering, and

$$
\phi(t)=\int_{0}^{t} 2 \pi f\left(t^{\prime}\right) d t^{\prime}+\phi_{c}
$$

is the phase of GW. For $h_{2}(t)$, i.e., the vacuum model, $a(t)$ and $f(t)$ are computed following a post-Newtonian approximation [40]. For $h_{1}(t)$, i.e., when there is gas, we add a term $1.5 f / T_{\text {gas }}$ to the equation of $\dot{f}$ to mimic the effect of gas drag.

To find the maximum FF, we explore the parameter space of $m_{1}$ and $\phi_{c}$ while keeping $q$ fixed to 0.7 , for simplicity. In a future work, we will complete the analysis by searching in the full parameter space of $\left(m_{1}, q, \phi_{c}\right)$. Our fiducial parameters are $\mathcal{M}=8.7 M_{\odot}, q=0.7, a(0)=0.002 \mathrm{AU}$, and $T_{\text {gas }} \simeq 10^{3}$ years. The values of the first three parameters are chosen such that in vacuum the $\mathrm{BBH}$ would merge on a timescale of $T_{\mathrm{gw}} \simeq 10^{4}$ years.

Figure 1 shows the resulting FF as a function of the LISA observing time, $T_{s}$. We find that FF is above 0.995 during the first 1.1 years of observation. The high FF means that the vacuum template gives a reasonable fit to the signal, even though it is the wrong template to use here. The best-fit $\mathcal{M}_{o}$ is about $36.94 M_{\odot}$, much larger than $\mathcal{M}$. This result agrees well with what we have envisioned in Section 2. 


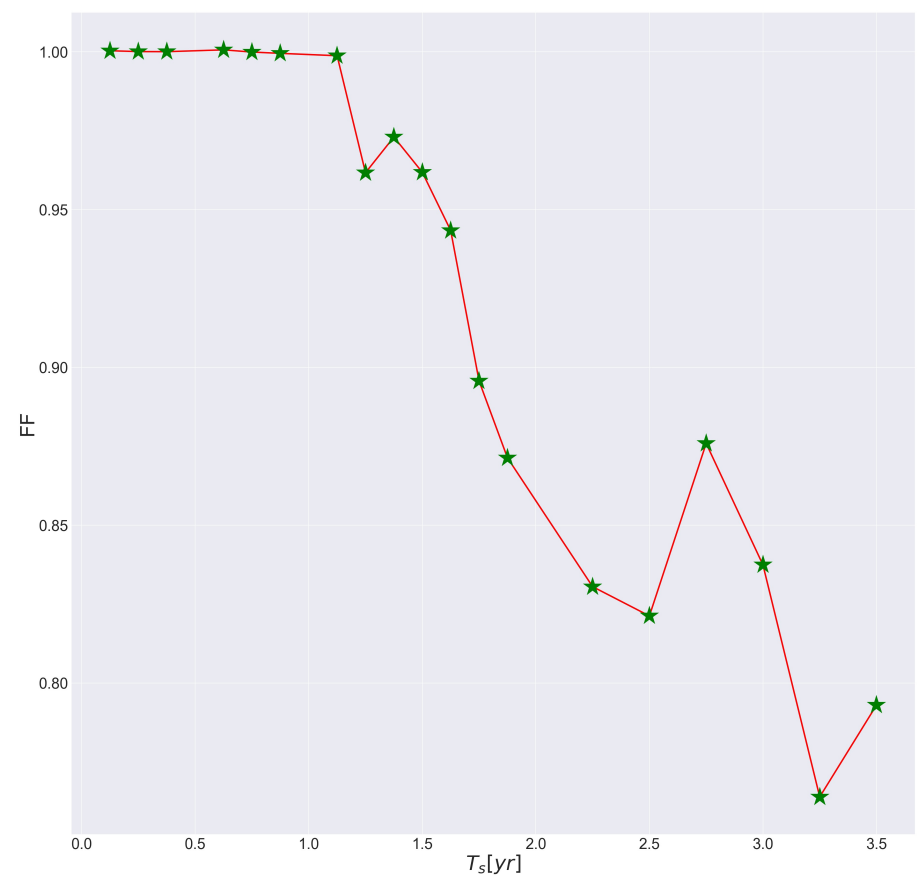

Figure 1. Fitting factor as a function of the observing time of GWs. The two waveforms which are compared here are $h_{1}$, the waveform of a low-mass BBH residing in a gaseous environment, and $h_{2}$, a high-mass $\mathrm{BBH}$ in vacuum. The input parameters are $\mathcal{M}=8.7 \mathrm{M}_{\odot}, q=0.7, a=0.002 \mathrm{AU}$, and $T_{\text {gas }} \simeq 10^{3}$ years. The best-fit chirp mass, corresponding to the highest FF, is $\mathcal{M}_{o} \simeq 37 M_{\odot}$.

When the observing time is longer than 1.1 years, the FF decays to a value below 0.995 . This result indicates that LISA would be able to distinguish the BBHs in gaseous environments from those in vacuum, given that the observing time is long enough. The exact time that is needed to reveal the difference depends on the parameters of the $\mathrm{BBH}$, as well as the properties of the surrounding gas. This issue deserves further investigation.

\section{Conclusions}

We have shown that the presence of gas around BBHs could affect the chirp signal and lead to a significant overestimation of the mass of the binaries. This effect is important for LISA observation but negligible for LIGO/Virgo sources. Our results have important implications for the future joint observation of BBHs using both ground and space-borne detectors [46,47].

Author Contributions: X.C. proposed the idea and methodology; Z.S. performed the parameter estimation using the matched filtering technique. Both authors contributed to the writing of the article.

Funding: This research was funded by the "985 Project" of Peking University and the National Science Foundation of China No. 11873022. X.C. is partly supported by the Strategic Priority Research Program of the Chinese Academy of Sciences, Grant Nos. XDB23040100 and No. XDB23010200.

Acknowledgments: The work is supported by the High-Performance Computing Platform of Peking University.

Conflicts of Interest: The authors declare no conflict of interest.

\section{References}

1. Schutz, B.F. Determining the Hubble constant from gravitational wave observations. Nature 1986, 323, 310, doi:10.1038/323310a0.

2. Chen, X.; Li, S.; Cao, Z. Mass-redshift degeneracy for the gravitational-wave sources in the vicinity of supermassive black holes. Mon. Not. R. Astron. Soc. 2019, 485, L141-L145, doi:10.1093/mnrasl/slz046. 
3. The LIGO Scientific Collaboration; The Virgo Collaboration. GWTC-1: A Gravitational-Wave Transient Catalog of Compact Binary Mergers Observed by LIGO and Virgo during the First, and Second Observing Runs. arXiv 2018, arXiv:1811.12907.

4. The LIGO Scientific Collaboration; The Virgo Collaboration. Binary Black Hole Population Properties Inferred from the First, and Second Observing Runs of Advanced LIGO and Advanced Virgo. arXiv 2018, arXiv:1811.12940.

5. McClintock, J.E.; Narayan, R.; Steiner, J.F. Black Hole Spin via Continuum Fitting and the Role of Spin in Powering Transient Jets. Space Sci. Rev. 2014, 183, 295-322, doi:10.1007/s11214-013-0003-9.

6. Corral-Santana, J.M.; Casares, J.; Muñoz-Darias, T.; Bauer, F.E.; Martínez-Pais, I.G.; Russell, D.M. BlackCAT: A catalogue of stellar-mass black holes in X-ray transients. Astron. Astrophys. 2016, 587, A61, doi:10.1051/0004-6361/201527130.

7. Abbott, B.P.; Abbott, R.; Abbott, T.D.; Abernathy, M.R.; Acernese, F.; Ackley, K.; Adams, C.; Adams, T.; Addesso, P.; Adhikari, R.X.; et al. Astrophysical Implications of the Binary Black-hole Merger GW150914. Astrophys. J. Lett. 2016, 818, L22, doi:10.3847/2041-8205/818/2/L22.

8. Amaro-Seoane, P.; Chen, X. Relativistic mergers of black hole binaries have large, similar masses, low spins and are circular. Mon. Not. R. Astron. Soc. 2016, 458, 3075-3082, doi:10.1093/mnras/stw503.

9. Stone, N.C.; Metzger, B.D.; Haiman, Z. Assisted inspirals of stellar mass black holes embedded in AGN discs: solving the 'final au problem'. Mon. Not. R. Astron. Soc. 2017, 464, 946-954, doi:10.1093/mnras/stw2260.

10. Bartos, I.; Kocsis, B.; Haiman, Z.; Márka, S. Rapid and Bright Stellar-mass Binary Black Hole Mergers in Active Galactic Nuclei. Astrophys. J. 2017, 835, 165, doi:10.3847/1538-4357/835/2/165.

11. McKernan, B.; Ford, K.E.S.; Bellovary, J.; Leigh, N.W.C.; Haiman, Z.; Kocsis, B.; Lyra, W.; Mac Low, M.M.; Metzger, B.; O’Dowd, M.; et al. Constraining Stellar-mass Black Hole Mergers in AGN Disks Detectable with LIGO. Astrophys. J. 2018, 866, 66, doi:10.3847/1538-4357/aadae5.

12. Secunda, A.; Bellovary, J.; Low, M.M.M.; Ford, K.E.S.; McKernan, B.; Leigh, N.W.C.; Lyra, W.; Sándor, Z. Orbital Migration of Interacting Stellar Mass Black Holes in Disks around Supermassive Black Holes. Astrophys. J. 2019, 878, 85. doi:10.3847/1538-4357/ab20ca.

13. Broadhurst, T.; Diego, J.M.; Smoot, George, I. Reinterpreting Low Frequency LIGO/Virgo Events as Magnified Stellar-Mass Black Holes at Cosmological Distances. arXiv 2018, arXiv:1802.05273.

14. Smith, G.P.; Jauzac, M.; Veitch, J.; Farr, W.M.; Massey, R.; Richard, J. What if LIGO's gravitational wave detections are strongly lensed by massive galaxy clusters? Mon. Not. R. Astron. Soc. 2018, 475, 3823-3828, doi:10.1093/mnras/sty031.

15. Hannuksela, O.A.; Haris, K.; Ng, K.K.Y.; Kumar, S.; Mehta, A.K.; Keitel, D.; Li, T.G.F.; Ajith, P. Search for Gravitational Lensing Signatures in LIGO-Virgo Binary Black Hole Events. Astrophys. J. 2019, 874, L2, doi:10.3847/2041-8213/ab0c0f.

16. Chen, X.; Han, W.B. Extreme-mass-ratio Inspirals Produced by Tidal Capture of Binary Black Holes. Commun. Phys. 2018, 1, 53, doi:10.1038/s42005-018-0053-0.

17. Han, W.B.; Chen, X. Testing general relativity using binary extreme-mass-ratio inspirals. Mon. Not. R. Astron. Soc. 2019, 485, L29-L33, doi:10.1093/mnrasl/slz021.

18. Amaro-Seoane, P.; Audley, H.; Babak, S.; Baker, J.; Barausse, E.; Bender, P.; Berti, E.; Binetruy, P.; Born, M.; Bortoluzzi, D.; et al. Laser Interferometer Space Antenna. arXiv 2017, arXiv:1702.00786.

19. Miller, M.C. Gravitational Radiation from Intermediate-Mass Black Holes. Astrophys. J. 2002, 581, 438-450, doi:10.1086/344156.

20. Sesana, A. Prospects for Multiband Gravitational-Wave Astronomy after GW150914. Phys. Rev. Lett. 2016, 116, 231102, doi:10.1103/PhysRevLett.116.231102.

21. Chen, X.; Amaro-Seoane, P. Revealing the Formation of Stellar-mass Black Hole Binaries: The Need for Deci-Hertz Gravitational-wave Observatories. Astrophys. J. Lett. 2017, 842, L2.

22. Seto, N. Strong gravitational lensing and localization of merging massive black hole binaries with LISA. Phys. Rev. D 2004, 69, 022002, doi:10.1103/PhysRevD.69.022002.

23. Sereno, M.; Jetzer, P.; Sesana, A.; Volonteri, M. Cosmography with strong lensing of LISA gravitational wave sources. Mon. Not. R. Astron. Soc. 2011, 415, 2773-2781, doi:10.1111/j.1365-2966.2011.18895.x.

24. Nakamura, T.T. Gravitational Lensing of Gravitational Waves from Inspiraling Binaries by a Point Mass Lens. Phys. Rev. Lett. 1998, 80, 1138-1141, doi:10.1103/PhysRevLett.80.1138. 
25. Takahashi, R.; Nakamura, T. Wave Effects in the Gravitational Lensing of Gravitational Waves from Chirping Binaries. Astrophys. J. 2003, 595, 1039-1051, doi:10.1086/377430.

26. Inayoshi, K.; Tamanini, N.; Caprini, C.; Haiman, Z. Probing stellar binary black hole formation in galactic nuclei via the imprint of their center of mass acceleration on their gravitational wave signal. Phys. Rev. D 2017, 96, 063014, doi:10.1103/PhysRevD.96.063014.

27. Meiron, Y.; Kocsis, B.; Loeb, A. Detecting Triple Systems with Gravitational Wave Observations. Astrophys. J. 2017, 834, 200, doi:10.3847/1538-4357/834/2/200.

28. Robson, T.; Cornish, N.J.; Tamanini, N.; Toonen, S. Detecting hierarchical stellar systems with LISA. Phys. Rev. D 2018, 98, 064012, doi:10.1103/PhysRevD.98.064012.

29. Chamberlain, K.; Moore, C.J.; Gerosa, D.; Yunes, N. Frequency-domain waveform approximants capturing Doppler shifts. Phys. Rev. D 2019, 99, 024025, doi:10.1103/PhysRevD.99.024025.

30. Wong, K.W.K.; Baibhav, V.; Berti, E. Binary radial velocity measurements with space-based gravitational-wave detectors. arXiv 2019, arXiv:1902.01402.

31. Hoang, B.M.; Naoz, S.; Kocsis, B.; Farr, W.M.; McIver, J. Detecting Supermassive Black Hole-induced Binary Eccentricity Oscillations with LISA. Astrophys. J. Lett. 2019, 875, L31, doi:10.3847/2041-8213/ab14f7.

32. Randall, L.; Xianyu, Z.Z. Observing Eccentricity Oscillations of Binary Black Holes in LISA. arXiv 2019, arXiv:1902.08604.

33. Fang, Y.; Huang, Q.G. Secular evolution of compact binaries revolving around a spinning massive black hole. Phys. Rev. D 2019, 99, 103005, doi:10.1103/PhysRevD.99.103005.

34. Antoni, A.; MacLeod, M.; Ramirez-Ruiz, E. The Evolution of Binaries in a Gaseous Medium: ThreeDimensional Simulations of Binary Bondi-Hoyle-Lyttleton Accretion. arXiv 2019, arXiv:1901.07572.

35. Loeb, A. Electromagnetic Counterparts to Black Hole Mergers Detected by LIGO. Astrophys. J. 2016, 819, L21, doi:10.3847/2041-8205/819/2/L21.

36. MacLeod, M.; Antoni, A.; Murguia-Berthier, A.; Macias, P.; Ramirez-Ruiz, E. Common Envelope Wind Tunnel: Coefficients of Drag and Accretion in a Simplified Context for Studying Flows around Objects Embedded within Stellar Envelopes. Astrophys. J. 2017, 838, 56, doi:10.3847/1538-4357/aa6117.

37. D'Orazio, D.J.; Loeb, A. Single progenitor model for GW150914 and GW170104. Phys. Rev. D 2018, 97, 083008, doi:10.1103/PhysRevD.97.083008.

38. Tagawa, H.; Saitoh, T.R.; Kocsis, B. Compact Object Mergers Driven by Gas Fallback. Phys. Rev. Lett. 2018, 120, 261101, doi:10.1103/PhysRevLett.120.261101.

39. Centrella, J.; Baker, J.G.; Kelly, B.J.; van Meter, J.R. Black-hole binaries, gravitational waves, and numerical relativity. Rev. Mod. Phys. 2010, 82, 3069-3119, doi:10.1103/RevModPhys.82.3069.

40. Cutler, C.; Flanagan, É.E. Gravitational waves from merging compact binaries: How accurately can one extract the binary's parameters from the inspiral waveform? Phys. Rev. D 1994, 49, 2658-2697, doi:10.1103/PhysRevD.49.2658.

41. Ostriker, E.C. Dynamical Friction in a Gaseous Medium. Astrophys. J. 1999, 513, 252-258.

42. Peters, P.C. Gravitational Radiation and the Motion of Two Point Masses. Phys. Rev. 1964, 136, 1224-1232, doi:10.1103/PhysRev.136.B1224.

43. Finn, L.S. Detection, measurement, and gravitational radiation. Phys. Rev. D 1992, 46, 5236-5249, doi:10.1103/PhysRevD.46.5236.

44. Lindblom, L.; Owen, B.J.; Brown, D.A. Model waveform accuracy standards for gravitational wave data analysis. Phys. Rev. D 2008, 78, 124020, doi:10.1103/PhysRevD.78.124020.

45. Kyutoku, K.; Seto, N. Concise estimate of the expected number of detections for stellar-mass binary black holes by eLISA. Mon. Not. R. Astron. Soc. 2016, 462, 2177-2183, doi:10.1093/mnras/stw1767.

46. Sesana, A. Multi-band gravitational wave astronomy: science with joint space- and ground-based observations of black hole binaries. J. Phys. Conf. Ser. 2017, 840, 012018.

47. Cutler, C.; Berti, E.; Holley-Bockelmann, K.; Jani, K.; Kovetz, E.D.; Larson, S.L.; Littenberg, T.; McWilliams, S.T.; Mueller, G.; Randall, L. What can we learn from multi-band observations of black hole binaries? Bull. Am. Astron. Soc. 2019, 51, 109,

(C) 2019 by the authors. Licensee MDPI, Basel, Switzerland. This article is an open access article distributed under the terms and conditions of the Creative Commons Attribution (CC BY) license (http://creativecommons.org/licenses/by/4.0/). 